\title{
ON RECURRENCES CONVERGING TO THE WRONG LIMIT IN FINITE PRECISION AND SOME NEW EXAMPLES*
}

\author{
SIEGFRIED M. RUMP ${ }^{\dagger}$
}

\begin{abstract}
In 1989, Jean-Michel Muller gave a famous example of a recurrence where, for particular initial values, the iteration over real numbers converges to a repellent fixed point, whereas finite precision arithmetic produces a different result, the attracting fixed point. We analyze recurrences in that spirit and remove a gap in previous arguments in the literature, that is, the recursion must be well defined. The latter is known as the Skolem problem. We identify initial values producing a limit equal to the repellent fixed point, show that in every $\varepsilon$-neighborhood of such initial values the recurrence is not well defined, and characterize initial values for which the recurrence is well defined. We give some new examples in that spirit. For example, the correct real result, i.e., the repellent fixed point, may be correctly computed in bfloat, half, single, double, formerly extended precision ( 80 bit format), binary 128 as well as many formats of much higher precision. Rounding errors may be beneficial by introducing some regularizing effect.
\end{abstract}

Key words. recurrences, rounding errors, IEEE-754, different precisions, bfloat, half precision (binary16), single precision (binary32), double precision (binary64), extended precision (binary128), multiple precision, Skolem problem, Pisot sequence

AMS subject classifications. 65G50, 11B37

1. Introduction. At the ICIAM 2019 conference in Valencia, the following famous recurrence was presented:

$$
x_{0}:=4, \quad x_{1}:=4.25, \quad \text { and } \quad x_{n+1}:=108-\left(815-1500 / x_{n-1}\right) / x_{n} .
$$

The true limit of this recurrence is $L=5$, whereas in double precision (binary64), the computed limit is 100 . This came as a surprise to the audience, so we decided to write this note giving the background and analysis of such recurrences.

The first example in that spirit is due to Muller [11]:

$$
x_{0}:=11 / 2, \quad x_{1}:=61 / 11, \quad \text { and } \quad x_{n+1}:=111-\left(1130-3000 / x_{n-1}\right) / x_{n} .
$$

The limit of the recurrence over the field of real numbers is $L=6$, whereas in double precision the limit is 100 . However, the initial value $x_{1}:=61 / 11$ is not representable in binary floating point format in any precision, so that for the input data stored in the computer, the limit 100 is correct.

The above example (1.1) was given by Kahan [9] together with an explanation of the behavior of the recurrence. In this example all input data including the initial values are representable in binary floating point format with at least 10 bits precision. In his book [12], Muller defines different initial values $x_{0}:=2, x_{1}:=-4$ for his recurrence (1.2), also representable in binary floating point format with at least 10 bits precision, with the same behavior of the recurrence as before.

The examples have in common the attracting fixed point $L=100$ together with a repellent fixed point $\beta=5$ or $\beta=6$, respectively. In Kahan's example, $x_{2}=\frac{76}{17}$, in Muller's first example, $x_{1}=\frac{61}{11}$, and in his second example, $x_{3}=\frac{347}{37}$. These values have in common that they are not representable in binary floating point format regardless of the precision. Replacing the initial value $\left(x_{k-1}, x_{k}\right)$ by the computed value $\left(x_{k-1}, \widetilde{x}_{k}\right)$, for $k=2,1,3$, respectively, it

${ }^{*}$ Received March 10, 2020. Accepted June 10, 2020. Published online on July 10, 2020. Recommended by Zdenek Strakoš.

${ }^{\dagger}$ Institute for Reliable Computing, Hamburg University of Technology, 21071 Hamburg, Germany and Visiting Professor at Waseda University, Tokyo 169-8555, Japan (rump@tuhh. de). 
follows that the recurrence over $\mathbb{R}$, if it is well defined, necessarily converges to the attracting fixed point $L=100$.

An implicit assumption for this assertion is that $x_{i} \neq 0$, for all $i \in \mathbb{N}_{0}$, otherwise the recurrence is not well defined. The problem of identifying the indices with an iterate being equal to zero for a linear recurrence is known as the Skolem problem [6, 14]. Instances of such problems are known to be NP-hard [2]. We will characterize the initial pairs $\left(x_{0}, x_{1}\right)$ for which such recurrences are well defined together with their limits.

We show that for every initial pair $\left(x_{0}, x_{1}\right)$ with the recurrence being well defined and converging to a repellent fixed point and any $\varepsilon$-neighborhood of $x_{1}$, there exists $x_{1}^{\prime}$ in this neighborhood such that the recurrence over $\mathbb{R}$ starting with $\left(x_{0}, x_{1}^{\prime}\right)$ produces $x_{n}=0$ for some $n \in \mathbb{N}$.

Moreover, it is suggested in the literature that, due to the fact that some iterates are not representable as floating point numbers, the iteration must converge to the attracting rather than the repellent fixed point. That may not be true for the floating point iteration due to "fortunate" rounding errors.

We give new explicit examples, starting with one where the correct value, i.e., the repellent fixed point, is computed in bfloat ${ }^{1}$, half precision, and single precision, but erroneous result are obtained in double precision (binary64) and extended precision (binary128). Other examples produce the correct limit—-the repellent fixed point—in much higher precision formats.

2. Analysis of recurrences. In the following we take a closer look at recurrences of type (1.1) or (1.2). We start the analysis in a more general setting and define

$$
x_{n+1}:=a+\left(b+c / x_{n-1}\right) / x_{n}, \quad \text { with } a, b, c \in \mathbb{R},
$$

for given initial values $\left(x_{0}, x_{1}\right) \in \mathbb{R}^{2}$. The auxiliary recurrence $y_{n+1}:=x_{n} y_{n}$, for $0 \leq n \in \mathbb{N}$ and $y_{0}:=1$, leads to

$$
\frac{y_{n+2}}{y_{n+1}}=a+\left(b+\frac{c y_{n-1}}{y_{n}}\right) \frac{y_{n}}{y_{n+1}}
$$

provided that $x_{n} \neq 0$, for $0 \leq n \in \mathbb{N}$, so that

$$
y_{n+2}=a y_{n+1}+b y_{n}+c y_{n-1}, \quad \text { for } 1 \leq n \in \mathbb{N} .
$$

Note that the linear recurrence (2.2) is always well defined. The characteristic polynomial is

$$
\chi(y)=y^{3}-a y^{2}-b y-c=:(y-\alpha)(y-\beta)(y-\gamma) .
$$

For simplicity we assume

$$
|\alpha|>|\beta|>|\gamma|>0 \quad \text { and } \quad \alpha, \beta, \gamma \in \mathbb{R} .
$$

For all examples above and the new examples to be presented later, this assumption is fulfilled. For given $y_{0}, y_{1}, y_{2}$, the recurrence (2.2) is characterized by a triple $(p, q, r) \in \mathbb{R}^{3}$ such that

$$
y_{n}=\alpha^{n} p+\beta^{n} q+\gamma^{n} r, \quad \text { for } 0 \leq n \in \mathbb{N} .
$$

The recurrence (2.1) is well defined, i.e., $x_{i} \neq 0$ for all $i \in \mathbb{N}_{0}$, if and only if $y_{i} \neq 0$ for all $i \in \mathbb{N}$. The initial value $y_{0}$ is only a scaling factor leading to the same recurrence (2.1). Using $y_{0}=p+q+r=1$, we can rewrite (2.5) into

$$
y_{n}=\left(\alpha^{n}-\gamma^{n}\right) p+\left(\beta^{n}-\gamma^{n}\right) q+\gamma^{n}, \quad \text { for } 0 \leq n \in \mathbb{N} .
$$

\footnotetext{
${ }^{1}$ bfloat [15] uses 16 bits like half precision but trades a larger exponent range against only 8 bit precision. It has been successfully used in deep learning and large scale networks [1,3]; see also [13].
} 
Thus, the initial values $\left(x_{0}, x_{1}\right)$ are coupled with $(p, q)$ by the linear system

$$
\left[\begin{array}{cc}
\alpha-\gamma & \beta-\gamma \\
\alpha^{2}-\gamma^{2} & \beta^{2}-\gamma^{2}
\end{array}\right]\left[\begin{array}{l}
p \\
q
\end{array}\right]=\left[\begin{array}{c}
x_{0}-\gamma \\
x_{0} x_{1}-\gamma^{2}
\end{array}\right]
$$

By assumption (2.4), the determinant $(\alpha-\beta)(\beta-\gamma)(\gamma-\alpha)$ is nonzero so that the linear system is solvable for all $\left(x_{0}, x_{1}\right)$.

The intention of the mentioned examples is that for the specified initial values $\left(x_{0}, x_{1}\right)$, the real recurrence $\left(x_{i}\right)$ as in (2.1) converges to the repellent fixed point $\beta$, whereas any perturbation of $\left(x_{0}, x_{1}\right)$ makes the recurrence (2.1) converge to the attracting fixed point $\alpha$, the root of largest absolute value. However, this includes the statement that the recurrence (2.1) is well defined for the given initial values $\left(x_{0}, x_{1}\right)$. Therefore, we next characterize the pairs $\left(x_{0}, x_{1}\right)$ for which this is true, i.e., the recurrence (2.1) is well defined and converges to $\beta$.

LEMmA 2.1. Let $x_{0}, x_{1} \in \mathbb{R}$ be given, and let the recurrence (2.1) with the characteristic polynomial (2.3) satisfy (2.4). Then (2.1) is well defined, and $x_{i} \rightarrow \beta$ if and only if

$$
\begin{array}{ll}
x_{0} \neq \gamma & \text { and } \\
x_{1}=\beta+\gamma-\beta \gamma / x_{0} & \text { and } \\
x_{0} \neq \gamma-\frac{\gamma^{n}(\beta-\gamma)}{\beta^{n}-\gamma^{n}} & \text { for all } n \geq 1 .
\end{array}
$$

REMARK 2.2. Note that the third condition implies that $x_{0} \neq 0$ for $n=1$, and for $n=2$ together with the second condition, also that $x_{1} \neq 0$. Also note that for the choice $x_{1}:=\beta+\gamma-\beta \gamma / x_{0}$, the iteration, being well defined or not, only depends on $x_{0}$.

Proof. By (2.6),

$$
\lim _{n \rightarrow \infty} x_{n}:= \begin{cases}\alpha & \text { if and only if } p \neq 0 \\ \beta & \text { if and only if } p=0, q \neq 0 \\ \gamma & \text { if and only if } p=q=0\end{cases}
$$

where the last case is equivalent to $x_{0}=x_{1}=\gamma$. Since (2.7) determines $p$ and $q$ uniquely, $\left(x_{i}\right)$ converges to $\beta$ if and only if $p=0$ and $q=\frac{x_{0}-\gamma}{\beta-\gamma} \neq 0$, which in turn is equivalent to

$$
x_{1}=\left(\left(\beta^{2}-\gamma^{2}\right) q+\gamma^{2}\right) / x_{0}=\beta+\gamma-\beta \gamma / x_{0} \quad \text { and } \quad x_{0} \neq \gamma \text {. }
$$

This means that if (2.1) is well defined, then $x_{i}$ converges to $\beta$ if and only if (2.8) and (2.9) hold true. The recurrence (2.1) is well defined if and only if $y_{n} \neq 0$ for all $n \geq 1$. If $p=0$ and $q \neq 0$, then this is by (2.6) equivalent to

$$
-\gamma^{n} \neq\left(\beta^{n}-\gamma^{n}\right) q=\frac{\left(\beta^{n}-\gamma^{n}\right)\left(x_{0}-\gamma\right)}{\beta-\gamma} \quad \text { for all } n \geq 1 .
$$

Therefore, the recurrence (2.1) is well defined if and only if (2.10) is true. This finishes the proof.

This shows that for $\left(x_{0}, x_{1}\right)$ on the hyperbola $H$ defined by $x_{1}=\beta+\gamma-\beta \gamma / x_{0}$, the recurrence $\left(x_{i}\right)$ is well defined and converges to $\beta$ except for infinitely many discrete points. The accumulation point of those gaps, determined by condition (2.10), is $x_{0}=\gamma$, the repellent fixed point with smallest absolute value. 
Next we show that the set of initial values $\left(x_{0}, x_{1}\right)$ with $x_{n}=0$ for some $n \in \mathbb{N}$, i.e., with a not well-defined recurrence, form hyperbolas $H_{n}$, and the limit $H$ of these hyperbolas is the hyperbola of initial values for which the recurrence converges to $\beta$ except for infinitely many discrete points.

LEMMA 2.3. Assume that the recurrence (2.1) with the characteristic polynomial (2.3) satisfies (2.4). For given $k \in \mathbb{N}$, denote by $Z_{k}$ the set of initial values $\left(x_{0}, x_{1}\right) \in \mathbb{R}^{2}$ with $x_{0} x_{1} \neq 0, x_{i} \neq 0$, for $0 \leq i<k$, and $x_{k}=0$.

Then there exists some $k_{0} \in \mathbb{N}$ such that for every $k \geq k_{0}$ the set $Z_{k}$ forms a hyperbola $H_{k}$, and for $k \rightarrow \infty$, the hyperbolas $H_{k}$ tend to the hyperbola $H$ of initial pairs with limit point $\beta$ of the recurrence, i.e., $x_{1}=\beta+\gamma-\beta \gamma / x_{0}$. If (2.4) is sharpened into $\alpha>\beta>\gamma>0$, then $k_{0}=2$.

Proof. Set $n:=k+1$. Let $\left(x_{0}, x_{1}\right)$ be given with $x_{0} x_{1} \neq 0, x_{i} \neq 0$, for $0 \leq i<k$, and $x_{n-1}=x_{k}=0$. Then $y_{n}=0$ and $y_{i} \neq 0$, for $0 \leq i<n$, and (2.6) implies

$$
M:=\left[\begin{array}{ccc}
\alpha-\gamma & \beta-\gamma & 0 \\
\alpha^{n}-\gamma^{n} & \beta^{n}-\gamma^{n} & 0 \\
\alpha^{2}-\gamma^{2} & \beta^{2}-\gamma^{2} & -x_{0}
\end{array}\right]\left[\begin{array}{c}
p \\
q \\
x_{1}
\end{array}\right]=\left[\begin{array}{c}
x_{0}-\gamma \\
-\gamma^{n} \\
-\gamma^{2}
\end{array}\right] .
$$

The determinant of the matrix is zero if and only if $(\alpha-\gamma)\left(\beta^{n}-\gamma^{n}\right)=(\beta-\gamma)\left(\alpha^{n}-\gamma^{n}\right)$, i.e.,

$$
f(\beta)=f(\alpha) \quad \text { for } f(x):=\frac{x^{n}-\gamma^{n}}{x-\gamma} .
$$

Hence, if $\alpha>\beta>\gamma>0$ and $x>\gamma$, then

$$
f(x)=x^{n-1}+x^{n-2} \gamma+\ldots+\gamma^{n-2} x+\gamma^{n-1}
$$

is strictly increasing, which shows that the determinant of the matrix in (2.12) is nonzero. The unique solution of the linear system is

$$
x_{1}=\frac{P-Q / x_{0}}{R} \quad \text { with }\left\{\begin{array}{l}
P=\alpha^{n}\left(\gamma^{2}-\beta^{2}\right)+\beta^{n}\left(\alpha^{2}-\gamma^{2}\right)+\gamma^{n}\left(\beta^{2}-\alpha^{2}\right), \\
Q=\alpha^{n} \beta \gamma(\gamma-\beta)+\beta^{n} \alpha \gamma(\alpha-\gamma)+\gamma^{n} \alpha \beta(\beta-\alpha), \\
R=\alpha^{n}(\gamma-\beta)+\beta^{n}(\alpha-\gamma)+\gamma^{n}(\beta-\alpha),
\end{array}\right.
$$

forming the desired hyperbola $H_{k}$. Note that $R=-\operatorname{det}(M) / x_{0}$, so that $x_{1}$ is well defined for $x_{0} \neq 0$. With (2.4) but without the assumption $\alpha>\beta>\gamma>0$, the determinant

$$
x_{0}\left(\alpha^{n}(\beta-\gamma)+\beta^{n}(\gamma-\alpha)+\gamma^{n}(\alpha-\beta)\right)
$$

of the matrix in (2.12) may vanish as, for example, for $\alpha=-7, \beta=5, \gamma=2$, and $n=3$. In this case, $x_{3} \neq 0$ for almost all values of $x_{0}$, and the hyperbola shrinks to a point. However, for large $n$ the determinant tends to $x_{0} \alpha^{n}(\beta-\gamma)$ by (2.4), and the linear system (2.12) is solvable for large enough $n$, thus the assertion remains valid.

For large enough $n$, the recurrence is not well defined on the hyperbola

$$
R^{(n)} x_{1}=P^{(n)}-Q^{(n)} / x_{0} .
$$

For $n \rightarrow \infty$,

$$
P^{(n)} \rightarrow \alpha^{n}\left(\gamma^{2}-\beta^{2}\right), \quad Q^{(n)} \rightarrow \alpha^{n} \beta \gamma(\gamma-\beta), \quad \text { and } \quad R^{(n)} \rightarrow \alpha^{n}(\gamma-\beta),
$$



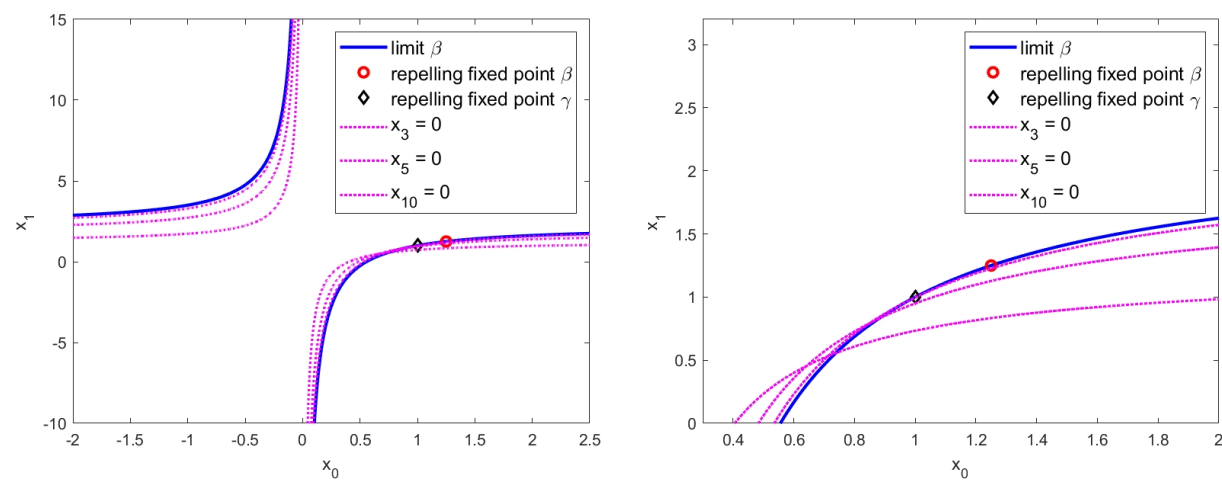

FIG. 2.1. Hyperbolas with limit $\beta$ and initial values producing a not well-defined recurrence.

which asymptotically corresponds to the hyperbola

$$
x_{1}=\frac{\gamma^{2}-\beta^{2}-\beta \gamma(\gamma-\beta) / x_{0}}{\gamma-\beta}=\beta+\gamma-\beta \gamma / x_{0} .
$$

This is the hyperbola $H$ of initial values $\left(x_{0}, x_{1}\right)$ yielding the limit point $\beta$.

In Figure 2.1 the hyperbola with initial values $\left(x_{0}, x_{1}\right)$ converging to the limit $\beta$ is displayed together with hyperbolas $H_{k}$ of initial values producing a not well-defined recurrence. In order to produce a better graph, we use a recurrence with roots of the characteristic polynomial close together, namely $\alpha=1.5, \beta=1.25$, and $\gamma=1$. Note that this does not change the qualitative behavior. The plot at the right-hand side in Figure 2.1 is a close-up near the unique initial values $\left(x_{0}, x_{1}\right)=(\gamma, \gamma)$, which yields convergence to the smallest root $\gamma$. In fact, the recurrence is entirely stationary in this case.

The previous Lemma 2.3 and in particular (2.13) imply that in every $\varepsilon$-neighborhood of initial values $\left(x_{0}, x_{1}\right)$ with a well-defined recurrence converging to $\beta$, there exists a pair of initial values with a not well-defined recurrence.

COROLLARY 2.4. Let the recurrence (2.1) with characteristic polynomial (2.3) satisfy (2.4). Suppose that for given initial values $\left(x_{0}, x_{1}\right) \in \mathbb{R}^{2}$ the recurrence $\left(x_{i}\right)$ is well defined and converges to $\beta$. Then for every $0<\varepsilon \in \mathbb{R}$, there exists $x_{1}^{\prime} \in \mathbb{R}$ with $\left|x_{1}^{\prime}-x_{1}\right|<\varepsilon$ such that the recurrence with initial values $\left(x_{0}, x_{1}^{\prime}\right)$ is not well defined.

Now we can verify the claims about the recurrences mentioned at the beginning. For Kahan's example (1.1), the roots are $(\alpha, \beta, \gamma)=(100,5,3)$. The recurrence with initial value $x_{0}$ is not well defined if and only if

$$
x_{0}=4=\gamma-\frac{\gamma^{n}(\beta-\gamma)}{\beta^{n}-\gamma^{n}}=3-\frac{2 \cdot 3^{n}}{5^{n}-3^{n}}=3-\frac{2}{(5 / 3)^{n}-1} \quad \text { for some } n \geq 1 .
$$

This is obviously not possible, so taking $x_{1}:=\beta+\gamma-\beta \gamma / x_{0}=5+3-15 / 4=4.25$ implies $x_{i} \rightarrow 5=\beta$.

For Muller's example (1.2), the roots are $(\alpha, \beta, \gamma)=(100,6,5)$. The recurrence with initial value $x_{0}$ is not well defined if and only if

$$
x_{0}=\gamma-\frac{\gamma^{n}(\beta-\gamma)}{\beta^{n}-\gamma^{n}}=5-\frac{5^{n}}{6^{n}-5^{n}}=5-\frac{1}{(6 / 5)^{n}-1} \quad \text { for some } n \geq 1 .
$$




\section{ETNA}

Kent State University and

Johann Radon Institute (RICAM)

TABLE 2.1

Results for the recurrence (2.14).

\begin{tabular}{rrrrcr}
$\mathrm{n}$ & half & single & double & over $\mathbb{R}$ \\
\hline 0 & 2.5019531 & 2.4999428 & 2.4999428 & $109225 / 43691$ & $\approx 2.4999428$ \\
1 & 2.5000000 & 2.5001144 & 2.5001144 & $10923 / 4369$ & $\approx 2.5001144$ \\
2 & 2.5937500 & 2.4997749 & 2.4997711 & $27305 / 10923$ & $\approx 2.4997711$ \\
3 & 4.4375000 & 2.5005341 & 2.5004578 & $13655 / 5461$ & $\approx 2.5004578$ \\
4 & 28.5000000 & 2.5009155 & 2.4990846 & $6825 / 2731$ & $\approx 2.4990846$ \\
5 & 56.2812500 & 2.5449677 & 2.5018315 & $683 / 273$ & $\approx 2.5018315$ \\
6 & 58.8750000 & 3.4965782 & 2.4963397 & $1705 / 683$ & $\approx 2.4963397$ \\
7 & 59 & 19.3815498 & 2.5073315 & $855 / 341$ & $\approx 2.5073314$ \\
8 & 59 & 53.8727341 & 2.4853823 & $425 / 171$ & $\approx 2.4853801$ \\
9 & 59 & 58.7636375 & 2.5294639 & $43 / 17$ & $\approx 2.5294118$ \\
10 & 59 & 58.9898109 & 2.4430787 & $105 / 43$ & $\approx 2.4418605$ \\
11 & 59 & 58.9995804 & 2.6483768 & $55 / 21$ & $\approx 2.6190476$ \\
12 & 59 & 58.9999809 & 2.9301292 & $25 / 11$ & $\approx 2.2727273$ \\
13 & 59 & 59 & 16.0674950 & 3 & \\
14 & 59 & 59 & 50.7931126 & $5 / 3$ & $\approx 1.6666667$ \\
15 & 59 & 59 & 58.7463651 & 5 & \\
16 & 59 & 59 & 58.9764139 & 0 & \\
17 & 59 & 59 & 59.0000847 & & \\
$\ldots$ & $\ldots$ & $\ldots$ & & & \\
27 & 59 & 59 & 59.0000000 & & \\
28 & 59 & 59 & 59 & & \\
29 & 59 & 59 & 59 & &
\end{tabular}

This is obviously not possible for the original value $x_{0}=11 / 2$ given in [11]. For $x_{0}=2$ given in [12], the recurrence is well defined if

$$
\frac{1}{(6 / 5)^{n}-1} \neq 3 \quad \text { for all } n \geq 1,
$$

which is equivalent to $n \neq \frac{\log (4 / 3)}{\log (6 / 5)} \approx 1.58$. Hence $\left(x_{i}\right)$ is well defined and converges to the repellent fixed point $6=\beta$.

2.1. Camouflaged convergence. It may not be visible from a floating point iteration that an iteration is, in fact, not well defined. Consider

$$
x_{0}:=\frac{109225}{43691}, x_{1}:=\frac{10923}{4369} \quad \text { and } \quad x_{n+1}:=56.5+\left(160-737.5 / x_{n-1}\right) / x_{n} .
$$

The roots of the characteristic polynomial are $(\alpha, \beta, \gamma)=(59,-5,2.5)$ and satisfy (2.4), and the initial values $\left(x_{0}, x_{1}\right)$ satisfy $x_{1}=\beta+\gamma-\beta \gamma / x_{0}$. According to Lemma 2.1, the limit is $\beta$ if the recurrence is well defined.

The result for this recurrence computed in half, single, double, and infinite precision is given in Table 2.1, illustrating the convergence to the attracting fixed point $\alpha=59$. The recurrence is constructed such that $x_{0}=\gamma-\frac{\gamma^{n}(\beta-\gamma)}{\beta^{n}-\gamma^{n}}$ for $n=17$, so that by Lemma 2.1, the recurrence over real numbers is not well defined. This fact is camouflaged by the floating point iteration in half, single, and in double precision.

As for Muller's original example in [11], the initial values $\left(x_{0}, x_{1}\right)$ in example (2.14) are not representable in binary floating point format, so in that respect, the limit 59 computed in half, single, and double precision is correct. 
TABLE 3.1

Precision format used.

\begin{tabular}{lcc} 
name & precision in bits & exponent bits \\
\hline bfloat (truncated binary16) & 8 & 8 \\
half precision (binary16) & 11 & 5 \\
single precision (binary32) & 24 & 8 \\
double precision (binary64) & 53 & 11
\end{tabular}

One may ask whether pathological examples of a recurrence exist with $x_{0}, x_{1}$ being exactly representable in binary floating point in some precision and $x_{1}$ on the hyperbola $x_{1}=\beta+\gamma-\beta \gamma / x_{0}$, but $x_{0}=\gamma-\frac{\gamma^{n}(\beta-\gamma)}{\beta^{n}-\gamma^{n}}$ for some $n \geq 1$. In this case, the pair $\left(x_{0}, x_{1}\right)$ is one of the described gaps, i.e., the conditions (2.8) and (2.9) for convergence to $\beta$ are satisfied, but by (2.10), the sequence is not well defined. We neither found such an example nor could we prove that it does not exist.

3. Yet other pathological examples. We finally give some new examples where very small precision formats yield the correct result, whereas higher precision formats do not. We use the four precision formats as in Table 3.1, three of them according to the IEEE-754 [8] floating point standard.

The format "bfloat" decreases the precision of binary 16 in order to increase the exponent range. It is often called truncated binary 16 , however, we use this format in rounding to nearest. The middle column gives the precision $k$ in bits including the implicit 1 , so that $2^{-k}$ is the relative rounding error unit. First, consider the recurrence

$$
x_{0}:=-6, \quad x_{1}:=64, \quad \text { and } \quad x_{n+1}:=82-\left(1824-6048 / x_{n-1}\right) / x_{n} .
$$

All input data are exactly representable in 8 bits binary precision, hence in bfloat and therefore in all other precision formats. The roots of the characteristic equation are $(\alpha, \beta, \gamma)=$ $(42,36,4)$. Thus, $\beta+\gamma-\beta \gamma / x_{0}=36+4+144 / 6=64=x_{1}$. By Lemma 2.1, the recurrence is not well defined if and only if

$$
x_{0}=-6=\gamma-\frac{\gamma^{n}(\beta-\gamma)}{\beta^{n}-\gamma^{n}}=4-\frac{32}{9^{n}-1} \quad \text { for some } n \geq 1 \text {. }
$$

This is obviously not possible.

The results for the different precision formats are displayed in Table 3.2. Clearly, from the last column, we observe that the iteration over $\mathbb{R}$ converges to the correct limit $\beta=36$, the repellent fixed point. The second iterate $x_{2}=\frac{151}{4}$ is computed without rounding error in all mentioned precision formats, but the third iterate $x_{3}=\frac{5464}{151}$ is not representable in any binary precision format and therefore rounded into some $\tilde{x}_{3}$.

The question whether the iteration is well defined or not depends only on the first initial value. Hence the iteration over the real numbers starting with $\left(x_{2}, \widetilde{x}_{3}\right)$ is also well defined. Therefore, mathematically, the iteration with initial values $\left(x_{2}, \widetilde{x}_{3}\right)$ converges to the attracting fixed point $\alpha=42$. However, due to "beneficial" rounding errors, the recurrence in bfloat, half, and single precision converges to the correct value, the repellent fixed point $\beta=36$.

When displayed as an integer (without trailing zeros) in Table 3.2, the value of the recurrence is equal to that integer. This happens in bfloat for $\widetilde{x}_{4}$, in half precision for $\widetilde{x}_{5}$, and in single precision for $\widetilde{x}_{9}$. The double precision recursion becomes stationary after some 444 iterations at $\widetilde{x}_{444} \approx 42-2.8 \cdot 10^{-14}$, close to the attracting fixed point $\alpha=42$.

It is sometimes suggested in the literature that, due to a rounding error in some iterate, the floating point iteration must converge to the attracting fixed point $\alpha=42$. In the example 


\begin{tabular}{|c|c|c|c|c|c|}
\hline \multicolumn{3}{|c|}{ Results for $x_{0}:=-6, x_{1}:=64$} & \multicolumn{3}{|c|}{ TABLE 3.2} \\
\hline $\mathrm{n}$ & bfloat & half & single & double & over $\mathbb{R}$ \\
\hline 0 & -6 & -6 & -6 & -6 & -6.000000 \\
\hline 1 & 64 & 64 & 64 & 64 & 64.000000 \\
\hline 2 & 37.750000 & 37.750000 & 37.750000 & 37.750000 & 37.750000 \\
\hline 3 & 36.250000 & 36.187500 & 36.185429 & 36.185430 & 36.185430 \\
\hline 4 & 36 & 36.031250 & 36.020496 & 36.020498 & 36.020498 \\
\hline 5 & 36 & 36 & 36.002277 & 36.002276 & 36.002276 \\
\hline 6 & 36 & 36 & 36.000256 & 36.000253 & 36.000253 \\
\hline 7 & 36 & 36 & 36.000031 & 36.000028 & 36.000028 \\
\hline 8 & 36 & 36 & 36.000004 & 36.000003 & 36.000003 \\
\hline 9 & 36 & 36 & 36 & 36.000000 & 36.000000 \\
\hline 10 & 36 & 36 & 36 & 36.000000 & 36.000000 \\
\hline 167 & $\begin{array}{ll}\cdots & \\
& \end{array}$ & $\begin{array}{l}\cdots \\
\end{array}$ & $\begin{array}{ll}\cdots & \\
& \end{array}$ & $\begin{array}{c}\ldots \\
36.000456\end{array}$ & $\begin{array}{c}\ldots \\
36.000000\end{array}$ \\
\hline 168 & 36 & 36 & 36 & 36.000532 & 36.000000 \\
\hline 169 & 36 & 36 & 36 & 36.000620 & 36.000000 \\
\hline 217 & $\ldots$ & $\cdots$ & $\ldots$ & 36.867247 & 36.000000 \\
\hline $\begin{array}{l}217 \\
218\end{array}$ & $\begin{array}{l}50 \\
36\end{array}$ & $\begin{array}{l}36 \\
36\end{array}$ & $\begin{array}{l}36 \\
36\end{array}$ & $\begin{array}{l}36.86 / 24 / \\
36.987987\end{array}$ & $\begin{array}{l}36.000000 \\
36.000000\end{array}$ \\
\hline 219 & 36 & 36 & 36 & 37.121863 & 36.000000 \\
\hline & $\ldots$ & $\ldots$ & $\cdots$ & $\ldots$ & \\
\hline 296 & 36 & 36 & 36 & 41.999817 & 36.000000 \\
\hline 297 & 36 & 36 & 36 & 41.999843 & 36.000000 \\
\hline 298 & 36 & 36 & 36 & 41.999866 & 36.000000 \\
\hline & $\begin{array}{ll}\cdots & \\
& \end{array}$ & $\cdots$ & $\ldots$ & $\begin{array}{r}\cdots \\
42.000\end{array}$ & 00000 \\
\hline 443 & 36 & 36 & 36 & 42.000000 & 36.000000 \\
\hline 444 & 36 & 36 & 36 & 42.000000 & 36.000000 \\
\hline
\end{tabular}

above this was true for double precision (binary64) but not true for smaller precision formats because of beneficial rounding errors.

One might think that increasing the precision further should yield the same erroneous result, namely suggesting a convergence to the attracting fixed point $\alpha=42$. However, this is not true. Table 3.3 displays results of the recurrence (3.1) for different initial values $\left(x_{0}, x_{1}\right)$ and different precision formats. Beyond those in Table 3.1 we add $^{2}$ the 80-bit format, formerly called extended precision, with 64 bits precision computed using [7] and IEEE-754 quadruple (binary128) with 113 bits precision. Using (3.2) one verifies that the recurrences are well defined.

Numbers shown with a decimal point represent a floating point number very close to $\alpha$ or $\beta$, close to working precision; otherwise the displayed integer is the stationary point of the floating point iteration. Results in bold face indicate that the recurrence produces the correct limit, i.e., the repellent fixed point $\beta$.

The first line corresponds to the initial values used in Table 3.2. As can be seen, increasing the precision to 64 bits yields the correct results, but a further increase produces the wrong but

${ }^{2}$ Many thanks to Kai Torben Ohlhus for performing the calculations in higher precision using MPFR [7]. 


\section{ETNA}

Kent State University and

Johann Radon Institute (RICAM)

\begin{tabular}{|c|c|c|c|c|c|c|c|}
\hline$x_{0}$ & $x_{1}$ & 8 & 11 & 24 & 53 & 64 & 113 \\
\hline-6 & 64 & 36 & 36 & 36 & 42.0 & 36 & 42.0 \\
\hline-288 & 40.5 & 36 & 36 & 36 & 36.0 & 42 & 42.0 \\
\hline-0.5 & 328 & 36 & 36 & 36 & 36.0 & 42.0 & 36.0 \\
\hline-0.1875 & 808 & 36 & 36 & 36 & 36.0 & 42.0 & 42.0 \\
\hline 0.5625 & -216 & 36 & 36 & 36 & 42.0 & 42.0 & 42.0 \\
\hline 64 & 37.75 & 36 & 36 & 36 & 42.0 & 36.0 & 42.0 \\
\hline
\end{tabular}

expected result, i.e., the attracting fixed point $\alpha$. But this need not to be so. In the third line, all but 64 bit precision produces the correct result due to beneficial rounding errors.

In all examples up to now, the repellent fixed point $\beta$ is in $\mathbb{F}$. It was asked [10] by Masahide Kashiwagi from Waseda University, Tokyo, whether this is mandatory. The following final example shows that this is not the case. Consider

$$
x_{0}:=8, \quad x_{1}:=-31, \quad \text { and } \quad x_{n+1}:=1.5+\left(972+128 / x_{n-1}\right) / x_{n} .
$$

All input data are representable in 8 bit binary floating point form, i.e., in bfloat and higher precision formats, and the characteristic equation $y^{3}-1.5 y^{2}-972 y-128$ has the roots

$$
\alpha=32, \quad \beta=\frac{-61-\sqrt{3657}}{4} \approx-30.3683, \quad \gamma=\frac{-61+\sqrt{3657}}{4} \approx-0.1317 .
$$

Using Lemma 2.1 one verifies that the recurrence is well defined.

The repellent fixed point $\beta$ is obviously not in $\mathbb{F}$, and the best we can expect is a stationary point $\tilde{\beta}$ near $\beta$. We say that the recurrence converges numerically to $\beta$ in precision $k$ bits if the relative error between $\tilde{\beta}$ and $\beta$ is of the order of the relative rounding error unit $2^{-k}$.

Running the recurrence (3.3) in different precisions of $k$ bits, it converges numerically to the repellent fixed point $\beta$

$$
\text { for all } k \in\{8,9, \ldots, 227\} \text { except for } k=183 .
$$

For all the precision formats with

$$
k \in\{8,9, \ldots, 227\} \backslash\{162,169,177,183,194,197,198,200,214,222\},
$$

the stationary point was the rounded-to-nearest value of $\beta$ in the given precision. Note that this includes bfloat, half, single, double, and extended precision.

Moreover, we ran the recurrence in all precision formats from 8 up to 5000 bits. The accumulated percentage of precision formats $[8,9, \ldots, 5000]$ with numerical convergence to $\beta$ is displayed in the upper curve of the left graph of Figure 3.1. For example, in about $90 \%$ of all precision formats from 8 to 1000 , or in about $60 \%$ of all precision formats from 8 to 3000 , a numerical convergence to $\beta$ was observed. The lower curve is the accumulated percentage with a stationary point equal to the rounded-to-nearest value of $\beta$; this is true, for example, for about $50 \%$ of all precision formats from 8 to 3000 . The right graph shows the same result with a close-up into the precision formats with $k \in[8,9, \ldots, 250]$.

Even for very high precision, recurrences of type (2.1) may converge numerically to $\beta$. For example, executing (3.3) in 17721 bits precision produces a stationary point close to $\beta$ up to the working precision after some 2262 iterations. 
FIG. 3.1. Accumulated percentage of precision formats with numerical convergence to $\beta$.

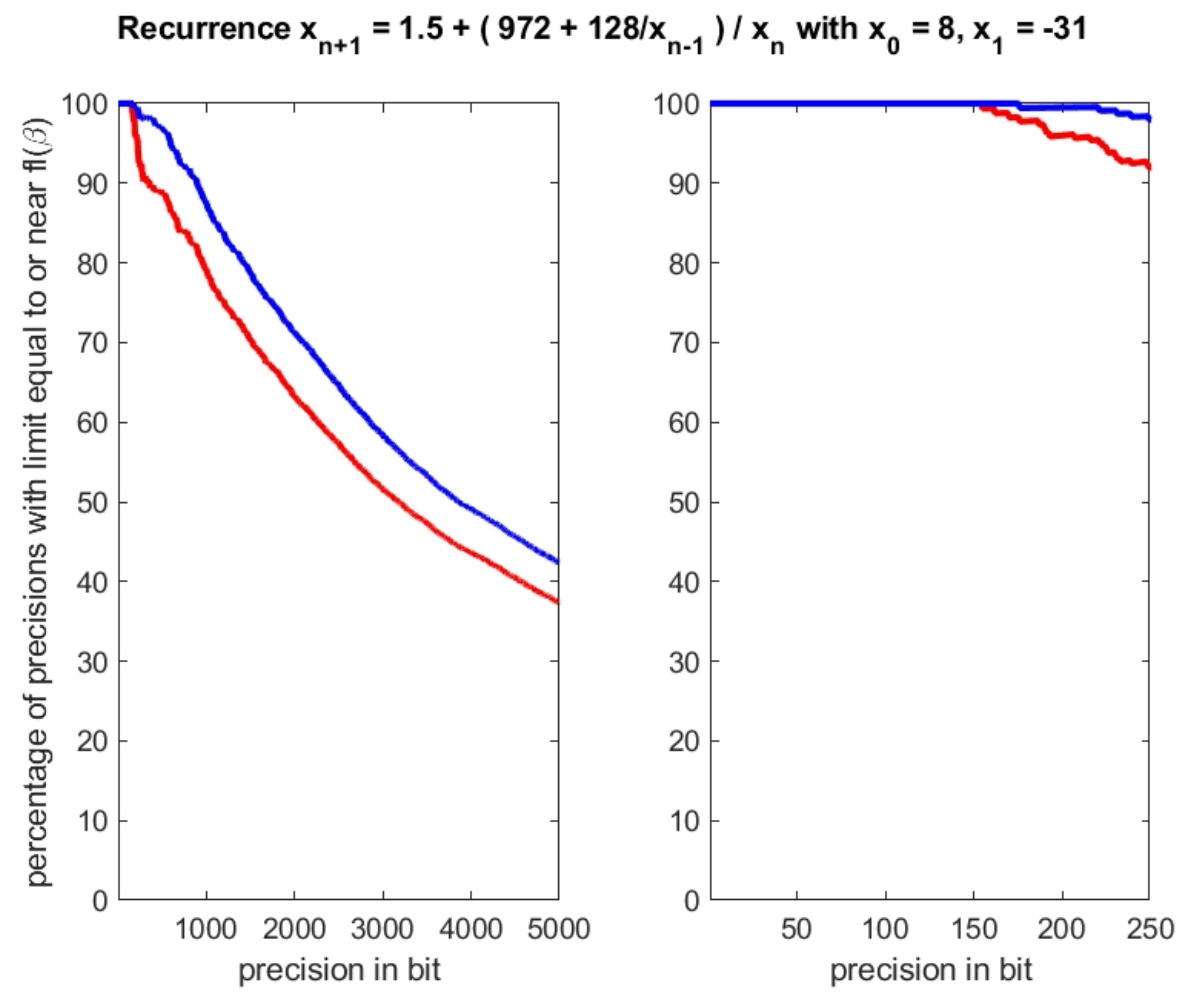

TABLE 3.4

Spectral radius of the Jacobian of (3.5) at the fixed points $\alpha, \beta$, and $\gamma$.

\begin{tabular}{c|ccc} 
recurrence & $\alpha$ & $\beta$ & $\gamma$ \\
\hline$(1.1)$ & 0.05 & 20 & 33.3 \\
$(1.2)$ & 0.06 & 16.7 & 20 \\
$(2.14)$ & 0.085 & 11.8 & 23.6 \\
$(3.1)$ & 0.86 & 1.17 & 10.5 \\
$(3.3)$ & 0.949 & 1.054 & 242.9
\end{tabular}

It has been pointed out by Masahide Kashiwagi [10] that such a behavior becomes clearer when looking at the stability of the recurrence at the fixed points. Writing the recurrence (2.1) as

$$
F(x, y):=\left[\begin{array}{c}
y \\
a+(b+c / x) / y
\end{array}\right]
$$

and evaluating the spectral radius of the Jacobian at the fixed points yields the results as in Table 3.4. The smaller the spectral radius for the attracting fixed point $\alpha$, the more we may expect a stable recurrence. Similarly, for a spectral radius for the repellent fixed point $\beta$ close to 1 , instabilities are more likely. This is particularly the case for the iterations (3.1) and even more for (3.3). 
We may add some interpretation of the results of the recurrence (3.3) in different precision formats: whether they are correct or not depends on the point of view. Executed over $\mathbb{R}$, the recurrence converges to the repellent fixed point $\beta$; in that respect the result is correct for all precision formats listed in (3.4) but incorrect for $k=183$. However, $x_{2}=\frac{-1883}{62}$ is not a binary floating point number in any precision but necessarily rounded into some $\tilde{x}_{2}$. The limit of the real recurrence (3.3) with initial values $x_{1}$ and $\tilde{x}_{2}$ is the attracting fixed point $\alpha$, and in that respect the result for precision $k=183$ is correct, but for all other precision format listed in (3.4) it is incorrect.

4. Conclusion. An analysis of recurrences based on Muller's initial example (1.2) is presented. Necessary and sufficient conditions are given for the recurrence being well defined and for convergence to a repellent fixed point. It is shown that in every $\varepsilon$-neighborhood of initial values $\left(x_{0}, x_{1}\right)$ that lead to convergence to a repellent fixed point, there exist initial values $\left(x_{0}, \tilde{x}_{1}\right)$ producing a not well-defined recurrence.

New recurrences are presented converging (correctly) to a repellent fixed point for smaller precision formats such bfloat, half, single, and double, but (incorrectly) not for higher precision formats. Another example shows convergence to a repellent fixed point even for very high precision like 5000 bits and more. As a result, this is another confirmation of the fact that rounding errors may be beneficial, and floating point may have a regularizing effect [4].

Acknowledgment. Many thanks to Florian Bünger, who read the manuscript very carefully and gave several valuable comments, and to Masahide Kashiwagi for fruitful discussions and suggestions. Also many thanks to Kai Torben Ohlhus for performing the calculations in higher precision. Moreover my thanks to an anonymous referee for his or her fruitful remarks and selfless work.

\section{REFERENCES}

[1] M. Abadi, A.Agarwal, P. Barham, E. Brevdo, Z. Chen, C. Citro, G. Corrado, A. Davis, J. Dean, M. Devin, S. Ghemawat, I. Goodfellow, A. Harp, G. Irving, M. ISARd, Y. Jia, R. Józefowicz, L. Kaiser, M. Kudlur, J. Levenberg, D. Mané, R. Monga, S. Moore, D. Murray, C. Olah, M. Schuster, J. Shlens, R. Steiner, I. Sutskever, K. Talwar, P. Tucker, V. Vanhoucke, V. Vasudevan, F. Viégas, O. Vinyals, P. Warden, M. Wattenberg, M. Wicke, Y. Yu, and $\mathrm{X}$. ZHENG, Tensorflow: large-scale machine learning on heterogeneous distributed systems, Preprint, CoRR, abs/1603.04467, 2016. https://arxiv.org/abs/1603.04467.

[2] V. D. BLONDEL, The presence of a zero in an integer recurrent sequence is NP-hard to decide, Linear Algebra Appl, 351/352 (2002), pp. 91-98.

[3] J. Dean, G. Corrado, R. Monga, K. Chen, D. Matthieu, M. Mao, M. Ranzato, A. Senior, P. TUCKER, K. YANG, Q. LE, AND A. NG, Large scale distributed deep networks, in Advances in Neural Information Processing Systems 25 (NIPS 2012), F. Pereira, C. J. C. Burges, L. Bottou, and K. Q. Weinberger, eds., NIPS Proceedings, Curran Associates, Red Hook, 2012, pp. 1223-1231.

[4] N. J. HIGHAM, A multiprecision world, SIAM News, October 2017.

[5] P. HoloborodKo, Multiprecision Computing Toolbox for MATLAB 4.6.4.13348, Advanpix LLC., Yokohama, Japan, 2019.

[6] K. MAHLER, Eine arithmetische Eigenschaft der Taylorkoeffizienten rationaler Funktionen, Akad. Wetensch. Amsterdam, 38 (1935), pp. 50-60.

[7] L. Fousse, G. Hanrot, V. Lefèvre, P. PÉlissier, And P. Zimmermann, MPFR: A multiple-precision binary floating-point library with correct rounding, ACM Trans. Math. Software, 33 (2007), Art. 13, 15 pages.

[8] IEEE, IEEE Standard for Floating-Point Arithmetic 754-2008, The Institute of Electrical and Electronics Engineers, New York, August 2008.

[9] W. KAHAN, How futile are mindless assessments of roundoff in floating-point computations, Preprint, University of California, Berkeley, 2006.

https://people.eecs.berkeley.edu/ wkahan/Mindless.pdf

[10] M. KaSHIWAGI, Private communication, 2019. 
[11] J.-M. Muller, Arithmétique des Ordinateurs, Masson, Paris, 1989. https://hal-ens-lyon.archives-ouvertes.fr/ensl-00086707.

[12] J.M. Muller, N. Brunie, F. De Dinechin, C.-P. Jeannerod, M. Joldes, V. Lefevre, G. Melquiond, R. ReVol, AND S. Torres, Handbook of Floating-Point Arithmetic, 2nd ed., Birkhäuser, Cham, 2018.

[13] S. PRANESH, Low precision floating-point formats: the wild west of computer arithmetic, SIAM News, November 2019.

[14] T. SKOLEM, Einige Sätze über gewisse Reihenentwicklungen und exponentiale Beziehungen mit Anwendung auf diophantische Gleichungen, Oslo Vid. Akad. Skrifter I, 6 (1933), pp. 76-89.

[15] G. Tagliavini, S. Mach, D. Rossi, A. MarongiU, and L. Benini A transprecision floating-point platform for ultra-low power computing, Preprint, CoRR, abs/1711.10374, 2017.

https://arxiv.org/abs/1711.10374 